\title{
Molecular Network Analysis of the Urinary Proteome of Alzheimer's Disease Patients
}

\author{
Yumi Watanabe $^{a}$ Yoshitoshi Hirao $^{b}$ Kensaku Kasugac \\ Takayoshi Tokutake $^{d}$ Yuka Semizu $^{a}$ Kaori Kitamura ${ }^{a}$ Takeshi Ikeuchic \\ Kazutoshi Nakamura ${ }^{a}$ Tadashi Yamamoto ${ }^{b}$ \\ ${ }^{a}$ Division of Preventive Medicine, Niigata University Graduate School of Medical and Dental \\ Sciences, Niigata, Japan; b Biofluid Biomarker Center, Niigata University, Niigata, Japan; \\ 'Department of Molecular Genetics, Brain Research Institute, Niigata University, Niigata, \\ Japan; d Department of Neurology, Brain Research Institute, Niigata University, Niigata, \\ Japan
}

\section{Keywords}

Alzheimer's disease $\cdot$ Urine $\cdot$ Label-free mass spectrometry $\cdot$ Case-control study · Proteomics

\begin{abstract}
Background/Aims: The identification of predictive biomarkers for Alzheimer's disease (AD) from urine would aid in screening for the disease, but information about biological and pathophysiological changes in the urine of $A D$ patients is limited. This study aimed to explore the comprehensive profile and molecular network relations of urinary proteins in AD patients. Methods: Urine samples collected from 18 AD patients and 18 age- and sex-matched cognitively normal controls were analyzed by mass spectrometry and semiquantified with the normalized spectral index method. Bioinformatics analyses were performed on proteins which significantly increased by more than 2 -fold or decreased by less than 0.5 -fold compared to the control ( $p<0.05$ ) using DAVID bioinformatics resources and KeyMolnet software. Results: The levels of 109 proteins significantly differed between AD patients and controls. Among these, annotation clusters related to lysosomes, complement activation, and gluconeogenesis were significantly enriched. The molecular relation networks derived from these proteins were mainly associated with pathways of lipoprotein metabolism, heat shock protein 90 signaling, matrix metalloproteinase signaling, and redox regulation by thioredoxin. Conclusion: Our findings suggest that changes in the urinary proteome of AD patients reflect systemic changes related to $A D$ pathophysiology.


Watanabe et al.: Urinary Protein Profile of AD Patients

\section{Introduction}

Alzheimer's disease (AD) is a progressive neurodegenerative disease and the most common cause of dementia [1]. The main neuropathological changes associated with AD are extracellular accumulation of amyloid- $\beta$ plaques, intracellular accumulation of neurofibrillary tangles of $\tau$ protein, inflammation, and brain atrophy [1]. Unfortunately, no cure currently exists for AD. Recent studies have shown that brain changes associated with AD start more than a decade before the onset of clinical symptoms such as progressive memory deficits [2-5]. Thus, in order to reduce the incidence and prevalence of AD, it will be necessary to focus on the stage before clinical symptoms appear [6]. Accumulating evidence suggests that systemic metabolic dysfunction such as diabetes, vascular dysfunction, and systemic inflammation underlie the development of AD [7-9]. These systemic changes also precede the onset of clinical symptoms of the disease. The discovery of a panel of biomarkers that reflect these systemic changes and could therefore predict the development of AD would be valuable for screening those at risk.

Urine is one of the most preferred biofluids for biomarker discovery because urine collection is simple and noninvasive. Moreover, repeated urine sampling from the same individual is easy, as is collection of a sufficient volume for analysis compared to other biofluids $[10,11]$. Urine also contains systemic information since approximately $30 \%$ of urinary protein originates from plasma via blood filtration, with the remainder coming from the kidneys and the urinary tract $[10,12]$. With technological advances in mass spectrometry (MS), MS-based proteomics has been used to identify a large number of proteins belonging to the urinary proteome $[11,13]$. While the discovery of predictive biomarkers for AD from urine would be highly beneficial, information regarding biological and pathophysiological changes in the urine of $\mathrm{AD}$ patients is currently limited. In the present study, urinary proteomes of $\mathrm{AD}$ patients and cognitively normal elderly controls were compared to explore the comprehensive profile and molecular-network relations of the urinary proteome of AD patients.

\section{Materials and Methods}

\section{Participants and Classification}

AD patients were recruited from outpatients of Niigata University Hospital who were diagnosed with the disease based on criteria of the National Institute of Neurological and Communicative Disorders and Stroke AD and Related Disorders Association (NINCDSADRDA) and took the Mini-Mental State Examination (MMSE) [14] within a year of urine collection. The clinical characteristics of the AD group are summarized in online supplementary Table 1 (for all online suppl. material, see www.karger.com/doi/10.1159/000496100).

Age- and sex-matched cognitively normal controls (MMSE score >27) were selected from a subcohort (Sekikawa cohort) of the Murakami cohort, a population-based cohort study that targeted individuals aged between 40 and 74 years living in areas of the northern Niigata Prefecture (Murakami region) [15]. Participants provided urine samples at specific health checkups held by the national health insurance of Japan and underwent the MMSE within a year of urine collection.

\section{Urine Sample Collection and Laboratory Test}

Spot urine samples were obtained from participants. No restrictions on diet, drinking, or exercise were required prior to urine sampling. Urinary protein, urinary sugar, and occult blood were checked using urine test strips (Pretest 5bII [Wako, Japan] for AD samples and Hema-Combistix-long [Siemens Healthcare, Japan] for control samples). Urinary albumin and 
Table 1. Participant characteristics and results of the general urinalysis

\begin{tabular}{lllr}
\hline & AD $(n=18)$ & Control $(n=18)$ & $p$ value \\
\hline Age, years & $72.9 \pm 5.6$ & $72.8 \pm 5.2$ & 0.951 \\
Males, $n$ & 8 & 8 & 1.000 \\
MMSE points & $21.6 \pm 4.5$ & $28.8 \pm 0.7$ & $<0.001$ \\
Urinary albumin $^{\mathrm{a}}, \mu \mathrm{g} / \mathrm{mL}$ & $46.18 \pm 24.8(11)$ & $18.6 \pm 4.7(10)$ & 0.298 \\
Urinary creatinine, $\mathrm{mg} / \mathrm{dL}_{\text {Albumin/creatinine }}, \mathrm{mg} / \mathrm{gCr}$ & $106.4 \pm 13.8$ & $77.7 \pm 10.5$ & 0.107 \\
Results of the urine test strip $_{\text {Urinary protein level }}$ & $22.7 \pm 6.6$ & $22.4 \pm 6.0$ & 0.976 \\
Urinary blood level & $-(17), \pm(1)$ & $-(18)$ & 0.486 \\
Urinary glucose level & $-(16), 2+(28)$ & $-(14), 1+(1), 3+(1)$ & 0.486 \\
\hline
\end{tabular}

Results are presented as means \pm SD for continuous variables. Values in parentheses are numbers of patients. $p$ values were calculated using an unpaired $t$ test and Fisher's exact test for continuous variables and categorical variables, respectively. ${ }^{\text {a }}$ Undetectable $(<5 \mu \mathrm{g} / \mathrm{mL})$ in $7 \mathrm{AD}$ and 8 control group patients. The mean \pm SD of urinary albumin was calculated from values of detected samples. ${ }^{b}$ For calculation of this ratio, undetected albumin was substituted with $5 \mu \mathrm{g} / \mathrm{mL}$.

creatinine were measured by latex immunological nephelometry using a SPOTCHEM D-01 analyzer (SD-3810; Arkray Global Business, Inc. Japan). Urine samples were centrifuged at $1,000 \mathrm{~g}$ for $15 \mathrm{~min}$ and the supernatant was stored at $-20^{\circ} \mathrm{C}$ until use.

\section{MS and Semiquantification of Proteome Data}

Detailed methods for protein extraction, MS analysis, and semiquantification of proteome data can be found in the online supplementary material. In brief, urine proteins were precipitated by the methanol precipitation method, dissolved, and digested in solution by trypsin. Digested samples were purified using a C18 spin column and peptides (500 ng) were analyzed by liquid chromatography coupled to tandem MS (MS/MS). All MS and MS/MS spectrums were analyzed by MASCOT (v4.2; Matrix Science) for protein and peptide identification. Data were queried against the Uniprot/Swiss-Prot database. Identification of proteins and peptides was carried out with a significance threshold of $p<0.05$. The normalized spectral index $\left(\mathrm{SI}_{N}\right)$, a label-free quantification method [16], was used to compare protein abundance between different samples.

\section{Bioinformatics Analysis}

Gene enrichment analysis was performed using functional annotation clustering of DAVID Bioinformatics Resources 6.8 (https://david.ncifcrf.gov/home.jsp) [17, 18]. Molecular network analysis was performed using KeyMolnet software (KM Data, Tokyo, Japan) [19]. Details of the KeyMolnet analysis are provided in the online supplementary material.

\section{Statistical Analysis}

Statistical analysis was performed using SAS $^{\circledR}$ Studio 3.7 software (SAS Institute Inc., Cary, NC, USA). Means of protein abundance were compared using a $t$ test with Welch's correction $(\alpha=0.05)$. Pearson's $\rho(r)$ was used to assess expression-level correlations of proteins among and between AD and control groups. Multiple comparisons were accounted for by using a false discovery rate adjustment $(\mathrm{q}=0.05)$. Graphs were prepared using GraphPad software (GraphPad Prism version 7.0a for Mac; La Jolla, CA, USA). 


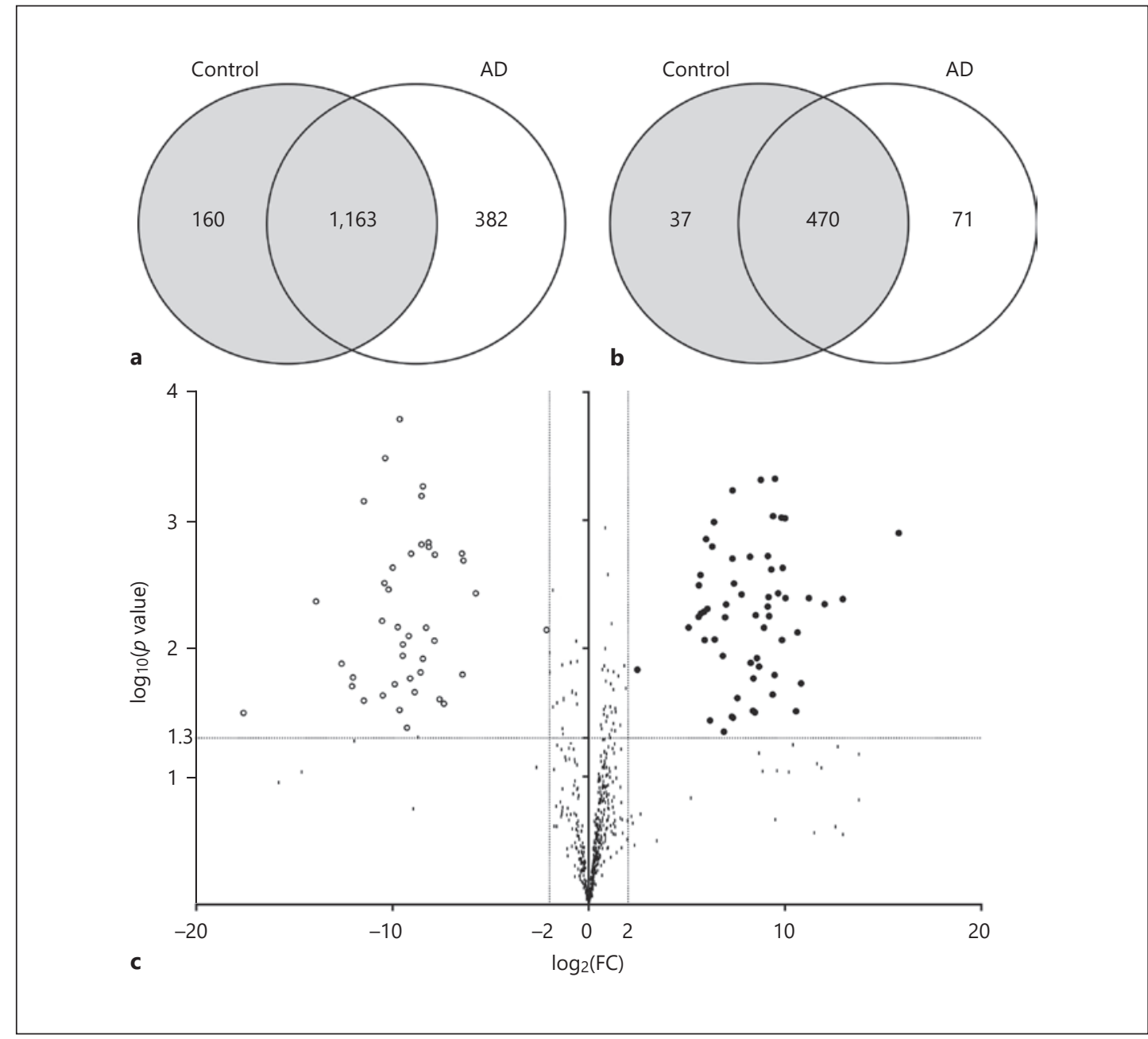

Fig. 1. Venn diagrams of all of the identified proteins (a) and proteins identified in at least 9 samples of either group (b). c Volcano plot displaying differentially expressed proteins between the AD and control groups. The $x$-axis displays the $\log 2$-fold change $(\mathrm{FC})$ of the mean $\mathrm{SI}_{\mathrm{N}}$ value between the groups, while the $y$-axis corresponds to the absolute value of log 10 ( $p$ value) of the $t$ test with Welch's correction. Closed black circles represent the 73 increased proteins in the $\mathrm{AD}$ group $(\mathrm{FC}>2, p<0.05)$ and open circles represent the 36 decreased proteins in the $\mathrm{AD}$ group $(\mathrm{FC}<0.5, p<0.05)$.

\section{Results}

Protein Identification by Liquid Chromatography-MS/MS and Semiquantification by $S_{N}$

Urine samples were collected from $18 \mathrm{AD}$ patients ( 8 males and 10 females) and 18 cognitively normal (MMSE $>27$ points) controls selected from participants of the Murakami cohort in an age- and sex-matched manner. General characteristics of the participants and the results of general urinalysis are shown in Table 1.

For AD and control urine samples, $613.2 \pm 117.7$ and $589.7 \pm 87.3$ (mean \pm SD) proteins were identified, respectively. Of the total of 1,705 unique proteins identified, 382 and 160 proteins were uniquely identified in AD and control groups, respectively, and 1,163 proteins were shared between the 2 groups (Fig. 1a). For further analysis, 578 proteins identified in at least 9 samples of either group, excluding 28 keratin isoforms, were selected. Of these, 71 and 37 proteins were uniquely identified in AD and control groups, respectively, and 470 
Table 2. List of 109 significantly increased or decreased proteins

\begin{tabular}{|c|c|c|c|c|c|c|c|c|c|}
\hline \multirow[t]{2}{*}{$\begin{array}{l}\text { Accession } \\
\text { No. }\end{array}$} & \multirow[t]{2}{*}{$\begin{array}{l}\text { MW, } \\
\mathrm{kDa}\end{array}$} & \multirow[t]{2}{*}{ Protein description (gene name) } & \multirow{2}{*}{$\begin{array}{l}\log _{2} \mathrm{FC} \\
\text { (AD/ } \\
\text { control) }\end{array}$} & \multicolumn{2}{|c|}{ Mean $\mathrm{SI}_{\mathrm{N}}$ value } & \multicolumn{2}{|l|}{$\begin{array}{l}\text { Detected } \\
\text { cases, } n\end{array}$} & \multirow[t]{2}{*}{$\begin{array}{l}\text { Welch's } \\
p \text { value }\end{array}$} & \multirow[t]{2}{*}{ FDR } \\
\hline & & & & $\mathrm{AD}$ & control & $\begin{array}{l}\mathrm{AD} \\
(n=18)\end{array}$ & $\begin{array}{l}\text { control } \\
(n=18)\end{array}$ & & \\
\hline P02042 & 16.0 & Hemoglobin subunit $\delta$ (HBD) & -17.59 & 0.0001 & 19.7571 & 0 & 10 & 0.032 & 0.166 \\
\hline Q8N2U0 & 11.7 & Transmembrane protein 256 (TMEM256) & -10.53 & 0.0001 & 0.1478 & 0 & 9 & 0.006 & 0.068 \\
\hline A0AVF1 & 64.1 & Intraflagellar transport protein 56 (TTC26) & -10.49 & 0.0001 & 0.1440 & 0 & 12 & 0.023 & 0.138 \\
\hline P81605-2 & 12.4 & Isoform 2 of dermcidin (DCD) & -10.41 & 0.0001 & 0.1360 & 0 & 13 & 0.003 & 0.056 \\
\hline P06703 & 10.2 & Protein S100-A6 (S100A6) & -10.37 & 0.0001 & 0.1320 & $\mathbf{0}$ & 15 & 0.000 & 0.048 \\
\hline P54710 & 7.3 & Sodium/potassium-transporting ATPase subunit $\gamma$ (FXYD2) & -10.20 & 0.0001 & 0.1178 & 0 & 9 & 0.004 & 0.058 \\
\hline Q15485 & 34.0 & Ficolin-2 (FCN2) & -9.99 & 0.0001 & 0.1017 & 0 & 12 & 0.002 & 0.050 \\
\hline P20827 & 23.8 & Ephrin-A1 (EFNA1) & -9.88 & 0.0001 & 0.0941 & 0 & 10 & 0.019 & 0.123 \\
\hline P21926 & 25.4 & CD9 antigen(CD9) & -9.73 & 0.0001 & 0.0852 & 0 & 9 & 0.007 & 0.070 \\
\hline P01127 & 27.3 & Platelet-derived growth factor subunit B (PDGFB) & -9.64 & 0.0001 & 0.0796 & 0 & 9 & 0.030 & 0.164 \\
\hline P02656 & 10.8 & Apolipoprotein C-III (APOC3) & -9.63 & 0.0001 & 0.0793 & $\mathbf{0}$ & 12 & 0.000 & 0.048 \\
\hline 000241 & 43.2 & Signal-regulatory protein $\beta_{1}$ (SIRPB1) & -9.48 & 0.0001 & 0.0714 & 0 & 12 & 0.011 & 0.095 \\
\hline Q7LBR1 & 22.1 & Charged multivesicular body protein $1 \mathrm{~b}$ (CHMP1B) & -9.26 & 0.0001 & 0.0612 & 0 & 13 & 0.042 & 0.196 \\
\hline Q6UXB4 & 32.5 & C-type lectin domain family 4 member G (CLEC4G) & -9.17 & 0.0001 & 0.0576 & 0 & 12 & 0.008 & 0.077 \\
\hline Q96PP9 & 73.1 & Guanylate-binding protein 4 (GBP4) & -9.10 & 0.0001 & 0.0550 & 0 & 9 & 0.017 & 0.114 \\
\hline P36915 & 68.7 & Guanine nucleotide-binding protein-like 1 (GNL1) & -9.05 & 0.0001 & 0.0532 & $\mathbf{0}$ & 10 & 0.002 & 0.048 \\
\hline P61981 & 28.3 & $14-3-3$ protein $\gamma(Y W H A G)$ & -8.87 & 0.0001 & 0.0467 & 0 & 9 & 0.022 & 0.135 \\
\hline P09972 & 39.4 & Fructose-bisphosphate aldolase C (ALDOC) & -8.57 & 0.0001 & 0.0381 & 0 & 11 & 0.015 & 0.110 \\
\hline Q6UY14-3 & 118.7 & Isoform 3 of ADAMTS-like protein 4 (ADAMTSL4) & -8.52 & 0.0001 & 0.0366 & $\mathbf{0}$ & 14 & 0.001 & 0.048 \\
\hline Q00796 & 38.3 & Sorbitol dehydrogenase (SORD) & -8.52 & 0.0001 & 0.0368 & $\mathbf{0}$ & 12 & 0.002 & 0.048 \\
\hline 000592 & 58.6 & Podocalyxin (PODXL) & -8.45 & 0.0001 & 0.0350 & $\mathbf{0}$ & 12 & 0.001 & 0.048 \\
\hline Q6FHJ7 & 39.8 & Secreted frizzled-related protein 4 (SFRP4) & -8.45 & 0.0001 & 0.0349 & 0 & 10 & 0.012 & 0.099 \\
\hline Р02748 & 63.1 & Complement component C9 (C9) & -8.29 & 0.0001 & 0.0313 & 0 & 10 & 0.007 & 0.070 \\
\hline P08238 & 83.2 & HSP 90- $\beta$ (HSP90AB1) & -8.16 & 0.0001 & 0.0286 & $\mathbf{0}$ & 10 & 0.002 & 0.048 \\
\hline Q9Y3B3 & 25.2 & $\begin{array}{l}\text { Transmembrane emp24 domain-containing protein } 7 \\
\text { (TMED7) }\end{array}$ & -8.15 & 0.0001 & 0.0284 & $\mathbf{0}$ & 9 & 0.002 & 0.048 \\
\hline 014578 & 231.4 & Citron $\rho$-interacting kinase $(\mathrm{CIT})$ & -7.86 & 0.0001 & 0.0233 & 0 & 10 & 0.009 & 0.079 \\
\hline P07711 & 37.5 & Cathepsin L1 (CTSL) & -7.84 & 0.0001 & 0.0229 & $\mathbf{0}$ & 10 & 0.002 & 0.048 \\
\hline P16284 & 82.5 & Platelet endothelial cell adhesion molecule (PECAM1) & -6.46 & 0.0001 & 0.0088 & $\mathbf{0}$ & 10 & 0.002 & 0.048 \\
\hline P20774 & 33.9 & Mimecan (OGN) & -6.43 & 0.0001 & 0.0086 & 0 & 9 & 0.016 & 0.111 \\
\hline Q9NPY3 & 68.5 & Complement component C1q receptor (CD93) & -6.38 & 0.0001 & 0.0083 & $\mathbf{0}$ & 9 & 0.002 & 0.049 \\
\hline P16234 & 122.6 & Platelet-derived growth factor receptor $\alpha$ (PDGFRA) & -5.75 & 0.0001 & 0.0054 & 0 & 10 & 0.004 & 0.058 \\
\hline P25311 & 34.2 & Zinc- $\alpha$-2-glycoprotein (AZGP1) & -2.15 & 5.6354 & 25.0614 & 17 & 18 & 0.007 & 0.072 \\
\hline P00746 & 27.0 & Complement factor D (CFD) & -1.30 & 0.0796 & 0.1959 & 9 & 13 & 0.048 & 0.217 \\
\hline Q9NQ84-2 & 49.4 & $\begin{array}{l}\text { Isoform } 2 \text { of G-protein coupled receptor family C group } 5 \\
\text { member C (GPRC5C) }\end{array}$ & 1.06 & 0.2948 & 0.1411 & 18 & 18 & 0.049 & 0.217 \\
\hline Q6GTX8 & 31.4 & Leukocyte-associated immunoglobulin-like receptor 1 (LAIR1) & 1.10 & 3.4443 & 1.6068 & 18 & 18 & 0.029 & 0.163 \\
\hline P21810 & 41.6 & Biglycan (BGN) & 1.13 & 0.1803 & 0.0822 & 15 & 17 & 0.020 & 0.124 \\
\hline P11047 & 177.5 & Laminin subunit $\gamma-1$ (LAMC1) & 1.17 & 0.0121 & 0.0054 & 16 & 12 & 0.007 & 0.070 \\
\hline P07602 & 58.1 & Prosaposin (PSAP) & 1.17 & 0.3748 & 0.1664 & 18 & 18 & 0.033 & 0.166 \\
\hline P10643 & 93.5 & Complement component C7 (C7) & 1.17 & 4.3890 & 1.9449 & 18 & 18 & 0.045 & 0.206 \\
\hline P35241-5 & 71.0 & Isoform 5 of radixin (RDX) & 1.26 & 0.2582 & 0.1081 & 16 & 18 & 0.022 & 0.134 \\
\hline P33908 & 72.9 & Mannosyl-oligosaccharide 1,2- $\alpha$-mannosidase IA (MAN1A1) & 1.26 & 0.1469 & 0.0611 & 13 & 10 & 0.040 & 0.191 \\
\hline P10253 & 105.3 & Lysosomal $\alpha$-glucosidase (GAA) & 1.27 & 1.3006 & 0.5395 & 18 & 18 & 0.016 & 0.111 \\
\hline Q9H3G5 & 54.1 & Probable serine carboxypeptidase CPVL (CPVL) & 1.34 & 0.2208 & 0.0870 & 15 & 11 & 0.045 & 0.206 \\
\hline P07686 & 63.1 & Hexosaminidase subunit $\beta$ (HEXB) & 1.35 & 0.1201 & 0.0472 & 15 & 12 & 0.030 & 0.164 \\
\hline Q12860 & 113.2 & Contactin-1 (CNTN1) & 1.37 & 0.0319 & 0.0124 & 16 & 11 & 0.032 & 0.166 \\
\hline P0DJD8 & 42.0 & Pepsin A-3 (PGA3) & 1.51 & 4.3450 & 1.5285 & 18 & 17 & 0.017 & 0.114 \\
\hline P06744-2 & 64.3 & Isoform 2 of glucose-6-phosphate isomerase (GPI) & 1.61 & 0.0687 & 0.0224 & 14 & 11 & 0.041 & 0.194 \\
\hline Q16651 & 36.4 & Prostasin (PRSS8) & 1.81 & 0.8232 & 0.2355 & 18 & 18 & 0.014 & 0.106 \\
\hline P34059 & 58.0 & $\mathrm{~N}$-acetylgalactosamine-6-sulfatase (GALNS) & 1.90 & 0.0533 & 0.0143 & 14 & 12 & 0.021 & 0.132 \\
\hline Q5JS37 & 38.3 & NHL repeat-containing protein 3 (NHLRC3) & 2.48 & 0.0701 & 0.0126 & 12 & 10 & 0.015 & 0.109 \\
\hline Q5JRA6 & 213.6 & Melanoma inhibitory activity protein 3 (MIA3) & 5.09 & 0.0034 & 0.0001 & 12 & 0 & 0.007 & 0.070 \\
\hline P13591 & 94.5 & Neural cell adhesion molecule 1 (NCAM1) & 5.60 & 0.0048 & 0.0001 & 9 & 0 & 0.006 & 0.065 \\
\hline Q7Z7M0 & 302.9 & $\begin{array}{l}\text { Multiple epidermal growth factor-like domain protein } 8 \\
\text { (MEGF8) }\end{array}$ & 5.61 & 0.0049 & 0.0001 & 13 & 0 & 0.003 & 0.056 \\
\hline Q92859 & 159.9 & Neogenin (NEO1) & 5.69 & 0.0052 & 0.0001 & 12 & 0 & 0.003 & 0.052 \\
\hline Q6UX71 & 59.5 & Plexin domain-containing protein 2 (PLXDC2) & 5.71 & 0.0052 & 0.0001 & 11 & 0 & 0.005 & 0.065 \\
\hline Q9NZV1 & 113.7 & Cysteine-rich motor neuron 1 protein (CRIM1) & 5.88 & 0.0059 & 0.0001 & 9 & 0 & 0.005 & 0.065 \\
\hline Q92563 & 46.7 & Testican-2 (SPOCK2) & 5.91 & 0.0060 & 0.0001 & 12 & 0 & 0.009 & 0.079 \\
\hline P08253 & 73.8 & 72-kDa type IV collagenase (MMP2) & 5.98 & 0.0063 & 0.0001 & 10 & $\mathbf{0}$ & 0.001 & 0.048 \\
\hline
\end{tabular}


Table 2 (continued)

\begin{tabular}{|c|c|c|c|c|c|c|c|c|c|}
\hline \multirow[t]{2}{*}{$\begin{array}{l}\text { Accession } \\
\text { No. }\end{array}$} & \multirow[t]{2}{*}{$\begin{array}{l}\text { MW, } \\
\mathrm{kDa}\end{array}$} & \multirow[t]{2}{*}{ Protein description (gene name) } & \multirow{2}{*}{$\begin{array}{l}\log _{2} \mathrm{FC} \\
(\mathrm{AD} / \\
\text { control) }\end{array}$} & \multicolumn{2}{|c|}{ Mean $\mathrm{SI}_{\mathrm{N}}$ value } & \multicolumn{2}{|l|}{$\begin{array}{l}\text { Detected } \\
\text { cases, } n\end{array}$} & \multirow[t]{2}{*}{$\begin{array}{l}\text { Welch's } \\
p \text { value }\end{array}$} & \multirow[t]{2}{*}{ FDR } \\
\hline & & & & $\mathrm{AD}$ & control & $\begin{array}{l}\mathrm{AD} \\
(n=18)\end{array}$ & $\begin{array}{l}\text { control } \\
(n=18)\end{array}$ & & \\
\hline Q15375 & 112.0 & Ephrin type-A receptor 7 (EPHA7) & 6.05 & 0.0066 & 0.0001 & 10 & 0 & 0.005 & 0.063 \\
\hline $\mathrm{P} 16112$ & 250.0 & Aggrecan core protein (ACAN) & 6.18 & 0.0072 & 0.0001 & 9 & 0 & 0.037 & 0.179 \\
\hline P07357 & 65.1 & Complement component $\mathrm{C} 8 \alpha$ chain $(\mathrm{CBA})$ & 6.29 & 0.0078 & 0.0001 & 9 & $\mathbf{0}$ & 0.002 & 0.048 \\
\hline Q92954 & 151.0 & Proteoglycan 4 (PRG4) & 6.38 & 0.0084 & 0.0001 & 11 & $\mathbf{0}$ & 0.001 & 0.048 \\
\hline Q66K79-2 & 72.5 & Isoform 2 of carboxypeptidase $\mathrm{Z}$ (CPZ) & 6.42 & 0.0086 & 0.0001 & 9 & 0 & 0.009 & 0.079 \\
\hline 075787 & 39.0 & Renin receptor (ATP6AP2) & 6.83 & 0.0114 & 0.0001 & 9 & 0 & 0.011 & 0.095 \\
\hline 043405 & 59.4 & Cochlin $(\mathrm{COCH})$ & 6.89 & 0.0118 & 0.0001 & 9 & 0 & 0.045 & 0.206 \\
\hline 000622 & 42.0 & Protein CYR61 (CYR61) & 6.95 & 0.0124 & 0.0001 & 9 & 0 & 0.006 & 0.065 \\
\hline Q9UJ96 & 51.2 & $\begin{array}{l}\text { Potassium voltage-gated channel subfamily G member } 2 \\
\text { (KCNG2) }\end{array}$ & 7.00 & 0.0128 & 0.0001 & 9 & 0 & 0.005 & 0.060 \\
\hline P50897 & 34.2 & Palmitoyl-protein thioesterase 1 (PPT1) & 7.29 & 0.0157 & 0.0001 & 9 & 0 & 0.034 & 0.172 \\
\hline P55083-2 & 31.1 & Isoform 2 of microfibril-associated glycoprotein 4 (MFAP4) & 7.32 & 0.0159 & 0.0001 & 10 & $\mathbf{0}$ & 0.002 & 0.048 \\
\hline 075309 & 89.9 & Cadherin-16 (CDH16) & 7.33 & 0.0161 & 0.0001 & 12 & $\mathbf{0}$ & 0.001 & 0.048 \\
\hline Q08345-5 & 101.7 & $\begin{array}{l}\text { Isoform } 4 \text { of epithelial discoidin domain-containing receptor } 1 \\
\text { (DDR1) }\end{array}$ & 7.35 & 0.0164 & 0.0001 & 10 & 0 & 0.035 & 0.173 \\
\hline P35858-2 & 70.2 & $\begin{array}{l}\text { Isoform } 2 \text { of insulin-like growth factor-binding protein } \\
\text { complex acid labile subun }\end{array}$ & 7.40 & 0.0169 & 0.0001 & 10 & 0 & 0.003 & 0.056 \\
\hline Q8NI32-2 & 23.3 & $\begin{array}{l}\text { Isoform } 2 \text { of Ly6/PLAUR domain-containing protein } 6 \mathrm{~B} \\
\text { (LYPD6B) }\end{array}$ & 7.57 & 0.0189 & 0.0001 & 11 & 0 & 0.024 & 0.142 \\
\hline Q8N307 & 71.9 & Mucin-20 (MUC20) & 7.79 & 0.0221 & 0.0001 & 11 & 0 & 0.004 & 0.058 \\
\hline P55957-2 & 26.8 & Isoform 2 of $\mathrm{BH} 3$-interacting domain death agonist (BID) & 8.22 & 0.0299 & 0.0001 & 9 & $\mathbf{0}$ & 0.002 & 0.048 \\
\hline P34896 & 53.0 & Serine hydroxymethyltransferase, cytosolic (SHMT1) & 8.25 & 0.0304 & 0.0001 & 12 & 0 & 0.013 & 0.103 \\
\hline Q6UX73 & 45.4 & UPF0764 protein C16orf89 (C16orf89) & 8.37 & 0.0332 & 0.0001 & 11 & 0 & 0.031 & 0.164 \\
\hline Q13145 & 29.1 & BMP and activin membrane-bound inhibitor homolog (BAMBI) & 8.39 & 0.0335 & 0.0001 & 9 & 0 & 0.017 & 0.114 \\
\hline Q8IV08 & 54.7 & Phospholipase D3 (PLD3) & 8.47 & 0.0355 & 0.0001 & 12 & 0 & 0.032 & 0.166 \\
\hline $\mathrm{P} 17174$ & 46.2 & Aspartate aminotransferase, cytoplasmic (GOT1) & 8.51 & 0.0365 & 0.0001 & 9 & 0 & 0.006 & 0.065 \\
\hline P23526 & 47.7 & Adenosylhomocysteinase (AHCY) & 8.57 & 0.0381 & 0.0001 & 9 & 0 & 0.012 & 0.098 \\
\hline P00491 & 32.1 & Purine nucleoside phosphorylase (PNP) & 8.68 & 0.0410 & 0.0001 & 9 & 0 & 0.014 & 0.106 \\
\hline Q9BRK5 & 41.8 & 45-kDa calcium-binding protein (SDF4) & 8.77 & 0.0437 & 0.0001 & 14 & $\mathbf{0}$ & 0.001 & 0.048 \\
\hline P10092 & 13.7 & Calcitonin gene-related peptide 2 (CALCB) & 8.93 & 0.0488 & 0.0001 & 10 & 0 & 0.007 & 0.070 \\
\hline Q9H444 & 24.9 & Charged multivesicular body protein $4 \mathrm{~b}(\mathrm{CHMP} 4 \mathrm{~B})$ & 9.12 & 0.0556 & 0.0001 & 12 & 0 & 0.005 & 0.062 \\
\hline P15121 & 35.8 & Aldose reductase (AKR1B1) & 9.13 & 0.0559 & 0.0001 & 13 & $\mathbf{0}$ & 0.002 & 0.048 \\
\hline 095445 & 21.2 & Apolipoprotein M (APOM) & 9.16 & 0.0572 & 0.0001 & 10 & 0 & 0.004 & 0.058 \\
\hline P41181 & 28.8 & Aquaporin-2 (AQP2) & 9.19 & 0.0585 & 0.0001 & 10 & 0 & 0.006 & 0.065 \\
\hline P01889 & 40.4 & HLA class I histocompatibility antigen, B-7 $\alpha$ chain (HLA-B) & 9.30 & 0.0631 & 0.0001 & 11 & 0 & 0.002 & 0.050 \\
\hline P60953 & 21.2 & Cell division control protein 42 homolog (CDC42) & 9.37 & 0.0660 & 0.0001 & 9 & 0 & 0.023 & 0.138 \\
\hline P78380 & 30.9 & Oxidized low-density lipoprotein receptor 1 (OLR1) & 9.39 & 0.0670 & 0.0001 & 12 & $\mathbf{0}$ & 0.001 & 0.048 \\
\hline P17050 & 46.5 & $\alpha-\mathrm{N}$-acetylgalactosaminidase (NAGA) & 9.47 & 0.0709 & 0.0001 & 12 & 0 & 0.016 & 0.111 \\
\hline P17936-2 & 32.2 & $\begin{array}{l}\text { Isoform } 2 \text { of insulin-like growth factor-binding protein } 3 \\
\text { (IGFBP3) }\end{array}$ & 9.49 & 0.0720 & 0.0001 & 11 & $\mathbf{0}$ & 0.001 & 0.048 \\
\hline P62873 & 37.4 & $\begin{array}{l}\text { Guanine nucleotide-binding protein } G(\mathrm{I}) / \mathrm{G}(\mathrm{S}) / \mathrm{G}(\mathrm{T}) \text { subunit } \\
\beta-1 \text { (GNB1) }\end{array}$ & 9.65 & 0.0801 & 0.0001 & 11 & 0 & 0.004 & 0.058 \\
\hline P16152 & 30.4 & Carbonyl reductase (NADPH) 1 (CBR1) & 9.82 & 0.0903 & 0.0001 & 11 & $\mathbf{0}$ & 0.001 & 0.048 \\
\hline P49441 & 44.0 & Inositol polyphosphate 1-phosphatase (INPP1) & 9.85 & 0.0921 & 0.0001 & 9 & 0 & 0.009 & 0.079 \\
\hline P15153 & 21.4 & Ras-related C3 botulinum toxin substrate 2 (RAC2) & 9.88 & 0.0942 & 0.0001 & 12 & 0 & 0.002 & 0.050 \\
\hline P29622 & 48.5 & Kallistatin (SERPINA4) & 10.00 & 0.1021 & 0.0001 & 13 & $\mathbf{0}$ & 0.001 & 0.048 \\
\hline P18669 & 28.8 & Phosphoglycerate mutase 1 (PGAM1) & 10.02 & 0.1038 & 0.0001 & 10 & 0 & 0.004 & 0.058 \\
\hline P31944 & 27.7 & Caspase-14 (CASP14) & 10.56 & 0.1510 & 0.0001 & 9 & 0 & 0.031 & 0.164 \\
\hline P10599 & 11.7 & Thioredoxin (TXN) & 10.64 & 0.1599 & 0.0001 & 10 & 0 & 0.008 & 0.073 \\
\hline P01225 & 14.7 & Follitropin subunit $\beta$ (FSHB) & 10.82 & 0.1804 & 0.0001 & 9 & 0 & 0.019 & 0.121 \\
\hline P62979 & 18.0 & Ubiquitin-40S ribosomal protein S27a (RPS27A) & 11.22 & 0.2393 & 0.0001 & 9 & 0 & 0.004 & 0.058 \\
\hline Q07654 & 8.6 & Trefoil factor 3 (TFF3) & 12.02 & 0.4156 & 0.0001 & 12 & 0 & 0.005 & 0.060 \\
\hline P04155 & 9.1 & Trefoil factor 1 (TFF1) & 12.95 & 0.7906 & 0.0001 & 13 & 0 & 0.004 & 0.058 \\
\hline P06312 & 13.4 & Ig $\kappa$ chain V-IV region (fragment) (IGKV4-1) & 15.80 & 5.6997 & 0.0001 & 12 & $\mathbf{0}$ & 0.001 & 0.048 \\
\hline
\end{tabular}

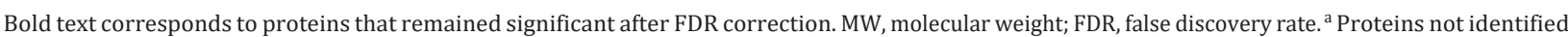
in certain samples were assumed to be at levels under the detection limit and thus were assigned a value that was half of the minimum $\mathrm{SI}_{\mathrm{N}}$ value (i.e., 0.0001 ). 
Table 3. Significantly enriched annotation clusters of significantly increased or decreased proteins ( $\mathrm{FC}>2$ or $<0.5, p<0.05$ ) calculated by DAVID Bioinformatics Resources 6.8

\begin{tabular}{|c|c|c|c|}
\hline Term & $p$ value & $\begin{array}{l}\text { Enrichment } \\
\text { score }\end{array}$ & Gene names \\
\hline \multicolumn{4}{|l|}{ Cluster 1} \\
\hline GO:0043202 lysosomal lumen & $<0.001$ & 4.25 & PSAP, PPT1, OGN, GAA, \\
\hline hsa04142: lysosome & $<0.001$ & & GALNS, BGN, HEXB, ACAN, \\
\hline GO:0005764 lysosome & 0.011 & & NAGA, CTSL, GOT1 \\
\hline \multicolumn{4}{|l|}{ Cluster 2} \\
\hline GO:0006957 complement activation, alternative pathway & $<0.001$ & 2.38 & LAMC1, C7, C8A, IGKV4-1, \\
\hline hsa05020: Prion diseases & $<0.001$ & & PNP, FCN2, C9, HLA-B, \\
\hline GO:0005579 membrane attack complex & 0.001 & & ITIH2, PRG4, HBD, NCAM1, \\
\hline G0:0006956 complement activation & 0.002 & & CFD \\
\hline GO:0072562 blood microparticle & 0.002 & & \\
\hline G0:0030449 regulation of complement activation & 0.014 & & \\
\hline hsa04610: complement and coagulation cascades & 0.018 & & \\
\hline GO:0006958 complement activation, classical pathway & 0.022 & & \\
\hline G0:0006955 immune response & 0.042 & & \\
\hline hsa05322: systemic lupus erythematosus & 0.296 & & \\
\hline \multicolumn{4}{|l|}{ Cluster 3} \\
\hline GO:0006094 gluconeogenesis & 0.002 & 1.7 & PGAM1, ALDOC, SHMT1, \\
\hline G0:0061621 canonical glycolysis & 0.011 & & GPI, GOT1 \\
\hline hsa01200: carbon metabolism & 0.013 & & \\
\hline G0:0006096 glycolytic process & 0.018 & & \\
\hline hsa01230: biosynthesis of amino acids & 0.022 & & \\
\hline hsa01130: biosynthesis of antibiotics & 0.092 & & \\
\hline hsa00010: glycolysis/gluconeogenesis & 0.102 & & \\
\hline
\end{tabular}

proteins were shared between the 2 groups (Fig. $1 \mathrm{~b}$ ). To estimate protein abundance, $\mathrm{SI}_{\mathrm{N}}$, a label-free quantification method, was used. Proteins that were not identified in certain samples were assumed to be at levels under the detection limit and thus they were assigned a value that was half of the minimum $\mathrm{SI}_{\mathrm{N}}$ value (i.e., 0.0001).

Welch's $t$ test identified 73 proteins that were significantly increased by more than 2 -fold and 36 proteins that were significantly decreased by less than 0.5 -fold in the AD group compared to the control group (Fig. 1c). The accession numbers, names, logarithm of the fold change ratio (AD/control) of the average $\mathrm{SI}_{\mathrm{N}}$ values, and molecular weights of proteins that were significantly increased or decreased are listed in Table 2. Twenty-four proteins remained significant after false discovery rate correction (bold in Table 2).

The expression-level correlations of proteins listed in Table 2 were analyzed in the AD group and the control group (online suppl. Tables 2A and B). Correlations were compared between $\mathrm{AD}$ and control groups (online suppl. Table 2C). Eleven correlations involving proteins (PSAP, RDX, C7, CPVL, LAIR1, GAA, GALNS, BGN, CNTN1, GPRC5C, and MAN1A1) were significant in both the AD group and the control group (online suppl. Table 2D), and 4 proteins (PSAP, GAA, GALNS, and BGN) were annotated to the lysosome.

\section{Bioinformatics Analysis}

To provide a broad overview of the identified proteins in each group, gene ontology (GO) analysis was performed using DAVID. The profiles of both groups had very similar distributions of GO annotations (online suppl. Fig. 1). To assess the functional significance of signifi- 
Watanabe et al.: Urinary Protein Profile of AD Patients

Table 4. List of the top 5 pathways contributing to the interrelation network and the AD-related network calculated by KeyMolnet

\begin{tabular}{llr}
\hline Rank & Pathway & Score \\
\hline \multicolumn{2}{l}{ Top 5} & pathways from the interrelation network \\
1 & HSP90 signaling pathway & 103.42 \\
2 & Lipoprotein metabolism & 86.74 \\
3 & Redox regulation by thioredoxin & 53.19 \\
4 & MMP signaling pathway & 45.63 \\
5 & Tetraspanin signaling pathway & 45.32 \\
\hline Top 5 & pathways from the AD-related network & \\
1 & Lipoprotein metabolism & 90.35 \\
2 & Transcriptional regulation by CREB & 88.81 \\
3 & HSP90 signaling pathway & 57.46 \\
4 & MMP signaling pathway & 47.19 \\
5 & Redox regulation by thioredoxin & 44.02
\end{tabular}

cantly increased or decreased proteins in the AD group, functional annotation clustering analysis with DAVID was performed using the GO database and KEGG pathway. Three annotation clusters related to lysosomes, complement activation, and gluconeogenesis were significantly enriched (Table 3; online suppl. Fig. 2).

To identify relationships between the molecular network of the urinary proteome and canonical pathway, an "interrelation" network search was performed using KeyMolnet. In the extracted molecular network, 18 pathways scored $>20$ and significantly contributed to the extracted network (online suppl. Table 3). The 5 pathways with the highest scores were the heat shock protein (HSP) 90 signaling pathway (score 104.424), lipoprotein metabolism (score 86.737), redox regulation by thioredoxin (score 53.188), the matrix metalloproteinase (MMP) signaling pathway (score 45.325), and the tetraspanin signaling pathway (score 45.319) (Table 4, upper panel). To extract molecular relations between AD-related molecules and the urinary proteome, a "start points and end points" network search was performed using KeyMolnet. We found that 71 proteins which were significantly increased or decreased in the urine proteome were associated with AD-related molecules directly or via an intermediate molecule (online suppl. Fig. 4), and 12 pathways significantly contributed to the extracted AD-related network (online suppl. Table 4). Of the 12 pathways, 4 of the top 5 were also listed among the top 5 pathways determined in the "interrelation" network search, i.e., lipoprotein metabolism (score 90.350), the HSP90 signaling pathway (score 57.456), the MMP signaling pathway (score, 47.188), and redox regulation by thioredoxin (score 44.021) (Table 4, lower panel).

\section{Discussion}

With recent advances in MS-based proteomics, urine has been used in biomarker studies of various diseases, not limited to renal and urogenital diseases, but also for nonurogenital diseases such as diabetes, osteoarthritis, cardiovascular disease, lung cancer, and other types of cancer [11, 20-30]. Many AD biomarker studies have used a proteomics approach, with most using CSF and blood but only a few using urine [31,32]. A comprehensive profile of the urinary proteome is important for urine biomarker discovery $[11,13]$. Therefore, in this study, we performed MS-based urine proteomics with label-free quantification, which offers a greater dynamic range and a wider proteome coverage compared to label-based methods [11], in order to gain a comprehensive view of the urinary proteome of AD patients. 
In this study, we compared the urinary proteome of $18 \mathrm{AD}$ patients and 18 age- and sexmatched cognitively normal elderly individuals. The average number of identified proteins in individual urine samples was 613 and 589 in the AD and control groups, respectively. This is comparable to previous reports $[13,33,34]$. Recent studies have found that systemic changes, such as insulin resistance, atherosclerosis, and increased inflammation underlie the development of AD [7-9]. Among the proteins significantly increased or decreased in the AD group compared to the control group, proteins related to lysosomes, the complement pathway, and gluconeogenesis were enriched. In the molecular network analysis, canonical pathways of lipoprotein metabolism, HSP90 signaling, MMP signaling, and redox regulation by thioredoxin significantly contributed to the molecular network of the urinary proteome and ADrelated molecules.

Lysosomes are major cellular organelles that digest and recycle all types of intracellular macromolecules and thus play a major role in protein homeostasis [35]. Previous studies have suggested the involvement of impaired lysosomal activity, including lysosomal enzyme malfunction, in the AD brain starting from an early stage of the disease [36-39]. In the present study, 7 of 11 lysosome-related proteins corresponded to lysosomal hydrolase. Previous studies have also reported increased activity of lysosomal glycohydrolases at the peripheral level in AD patients [40, 41].

Although most lysosome-related proteins were increased in AD urine, cathepsin L1 (CTSL) was not. Cathepsins are the most abundant lysosomal proteases and they have been implicated in neuronal death in AD patients [42]. Interestingly, CTSL activity is inhibited in the brains of aged animals [43]. Moreover, recent studies have found that CTSL functions as a key protease for the proteolytic processing of proneuropeptides into active neurotransmitters, and thus it is required for normal neurotransmission [44].

The complement system represents a key inflammatory pathway for the activation and execution of immune responses. Inflammatory responses in the brain are characteristic of AD pathology $[45,46]$. Recent studies have also revealed the occurrence of peripheral or systemic inflammation early in the development of $\operatorname{AD}[47,48]$.

Insulin is a key hormone that inhibits gluconeogenesis, and insulin resistance is a hallmark of type 2 diabetes [49]. Type 2 diabetes can cause mitochondrial dysfunction and promote an inflammatory response similar to that which triggers AD [50]. Epidemiological studies have found that the risk of $\mathrm{AD}$ is about 1.5-fold higher among people with diabetes than in the general population [51,52]. In the current study, we did not have sufficient information regarding the diabetic status of our participants, particularly the control group. However, the enrichment of proteins related to gluconeogenesis in the urine of $\mathrm{AD}$ patients is consistent with the known relationship between $\mathrm{AD}$ and diabetes.

Another protein listed in Table 2 that might participate in glucose metabolism is insulinlike growth factor-binding protein-3 (IGFBP3). IGFBP3 is a major binding protein of IGF-1 and several studies have reported its association with incident diabetes [53, 54]. Although results are inconsistent, alterations of circulating IGFBP3 levels in AD patients have been reported [55, 56].

Several recent studies have concluded that intrabrain vascular dysregulation is the earliest and strongest pathologic factor associated with late-onset AD [8, 57]. Atherosclerosis is a leading cause of vascular dysfunction, and it is the result of hyperlipidemia and lipid oxidation $[8,58]$. Thioredoxin is a major regulator of the cellular redox system that protects various cells from oxidative stress and is involved in atherogenesis [59]. According to one study, patients with atherosclerosis had an increased level of plasma thioredoxin-1 [60]. MMP are a large family of proteolytic enzymes and they have been implicated in the development and progression of atherosclerosis [61]. HSP90 is a molecular chaperone that prevents protein misfolding and aggregation [62]. HSP90 and HSP70 have been shown to 
exert their effects on atherosclerosis by influencing LDL metabolism, and the expression of HSP90 in atherosclerotic plaques has been associated with plaque instability [63].

Another vascular related protein that was significantly increased in AD urine is oxidized low-density lipoprotein receptor 1 (OLR1), which is also known as lectin-like oxidized lowdensity lipoprotein receptor-1 (LOX-1). OLR1/LOX-1 is a marker for atherosclerosis and it is induced by oxidative stress, inflammatory cytokines, and oxidized low-density lipoprotein [64]. Several studies have suggested an association of several SNP within OLR1 with AD [65].

Expression levels of several proteins in AD urine observed in the present study were inconsistent with previous reports. For example, S100A6 was significantly decreased in AD urine. In the brain, however, S100A6 has been reported to be upregulated in astrocytes of amyotrophic lateral sclerosis patients and in AD patients [66].

Although some studies have reported that hyperlipidemia is associated with AD pathogenesis, APOC3 was significantly decreased in AD urine. APOC3 is a major component of triglyceride-rich lipoproteins (chylomicrons and very low-density lipoprotein) and a minor component of high-density lipoprotein. Lin et al. [67] reported decreased levels of serum APOC3 with the progression of AD. Recent studies have reported that weight loss is a predictor of $\mathrm{AD}$ and may be related to the hypothalamic defects observed in $\mathrm{AD}$ patients $[68,69]$.

The present study has some limitations worth noting. First, the abundance of urinary proteins was estimated via a semiquantitative method and requires further validation by a quantitative method. Second, information regarding the comorbidities and renal function of participants was limited. Third, this study employed a cross-sectional case-control design. Further validation of our findings with a larger sample size and different populations is warranted. The above information notwithstanding, we were able to demonstrate differences in the urinary proteome of AD patients compared to cognitively normal controls and that the urinary proteome of AD patients reflects systemic changes that underlie AD pathophysiology. Further studies targeting earlier-stage AD patients or population-based prospective studies will help to clarify the potential of urine as a source of biomarkers for early screening of AD.

\section{Acknowledgement}

We thank all of the study participants and the following institutions for their contributions: the Murakami City Government and the Sekikawa Village Government. We also thank Dr. B. Xu, Dr. S. Saito, and all of the members of the Biofluid Biomarker Center, Niigata University, for their invaluable help.

\section{Statement of Ethics}

This study was approved by the human research ethics committee of Niigata University (approval No. 1836, 2015-2081). All of the patients or their guardians signed informed consent forms and all of the participants of the Murakami cohort were informed through an oral consent process.

\section{Disclosure Statement}

The authors declare that they have no conflicts of interests. 


\section{Funding Sources}

This work was supported in part by JSPS KAKENHI grant No. JP23249035, JP15H04782, and JP17K19799 (to K.N.) and JP16K09051 (to Y.W.); a grant from the SENSHIN Medical Research Foundation (to Y.W.); grant No. 18dm0107143 from the Japan Agency for Medical Research and Development (AMED) (to T.I.); and a grant from the Center of Innovation Program from MEXT (to T.Y.). The funders had no role in the study design, including collection, management, analysis, and interpretation of the data, the writing of this report, or the decision to submit this report for publication.

\section{Author Contributions}

Y. Watanabe, Y. Hirao, K. Kitamura., T. Yamamoto, and K. Nakamura contributed to the study concept and design. Y. Watanabe, K. Kitamura, Y. Semizu, T. Ikeuchi, K. Kasuga, T. Tokutake, and K. Nakamura contributed to acquisition of the data. Y. Watanabe, Y. Semizu, Y. Hirao, and T. Yamamoto contributed to analysis and interpretation of the data. Y. Watanabe and Y. Hirao drafted this paper. Y. Watanabe, Y. Hirao, K. Kitamura, T. Yamamoto, Y. Semizu, T. Ikeuchi, K. Kasuga, T. Tokutake, and K. Nakamura critically edited this paper for important intellectual content. Y. Watanabe and Y. Hirao contributed equally to this work. All of the authors approved the final version of this paper.

\section{References}

12018 Alzheimer's disease facts and figures. Alzheimers Dement. 2018 Mar;14(3):367-429.

2 Villemagne VL, Burnham S, Bourgeat P, Brown B, Ellis KA, Salvado O, et al.; Australian Imaging Biomarkers and Lifestyle (AIBL) Research Group. Amyloid $\beta$ deposition, neurodegeneration, and cognitive decline in sporadic Alzheimer's disease: a prospective cohort study. Lancet Neurol. 2013 Apr;12(4):357-67.

3 Reiman EM, Quiroz YT, Fleisher AS, Chen K, Velez-Pardo C, Jimenez-Del-Rio M, et al. Brain imaging and fluid biomarker analysis in young adults at genetic risk for autosomal dominant Alzheimer's disease in the presenilin 1 E280A kindred: a case-control study. Lancet Neurol. 2012 Dec;11(12):1048-56.

4 Bateman RJ, Xiong C, Benzinger TL, Fagan AM, Goate A, Fox NC, et al.; Dominantly Inherited Alzheimer Network. Clinical and biomarker changes in dominantly inherited Alzheimer's disease. N Engl J Med. 2012 Aug;367(9): 795-804.

5 Jack CR Jr, Lowe VJ, Weigand SD, Wiste HJ, Senjem ML, Knopman DS, et al.; Alzheimer's Disease Neuroimaging Initiative. Serial PIB and MRI in normal, mild cognitive impairment and Alzheimer's disease: implications for sequence of pathological events in Alzheimer's disease. Brain. 2009 May;132(Pt 5):1355-65.

6 Crous-Bou M, Minguillón C, Gramunt N, Molinuevo JL. Alzheimer's disease prevention: from risk factors to early intervention. Alzheimers Res Ther. 2017 Sep;9(1):71.

7 Graham WV, Bonito-Oliva A, Sakmar TP. Update on Alzheimer's Disease Therapy and Prevention Strategies. Annu Rev Med. 2017 Jan;68(1):413-30.

8 Wen SW, Wong CH. Aging- and vascular-related pathologies. Microcirculation. 2018 May; 50:e12463.

9 Xia X, Jiang Q, McDermott J, Han JJ. Aging and Alzheimer's disease: comparison and associations from molecular to system level. Aging Cell. 2018 Oct;17(5):e12802.

10 Decramer S, Gonzalez de Peredo A, Breuil B, Mischak H, Monsarrat B, Bascands JL, et al. Urine in clinical proteomics. Mol Cell Proteomics. 2008 Oct;7(10):1850-62.

11 Thomas S, Hao L, Ricke WA, Li L. Biomarker discovery in mass spectrometry-based urinary proteomics. Proteomics Clin Appl. 2016 Apr;10(4):358-70.

12 Beasley-Green A. Urine proteomics in the era of mass spectrometry. Int Neurourol J. 2016 Nov;20:S70-5.

13 Zhao M, Li M, Yang Y, Guo Z, Sun Y, Shao C, et al. A comprehensive analysis and annotation of human normal urinary proteome. Sci Rep. 2017 Jun;7(1):3024.

14 Folstein MF, Folstein SE, McHugh PR. "Mini-mental state". A practical method for grading the cognitive state of patients for the clinician. J Psychiatr Res. 1975 Nov;12(3):189-98.

15 Nakamura K, Takachi R, Kitamura K, Saito T, Kobayashi R, Oshiki R, et al. The Murakami Cohort Study of vitamin D for the prevention of musculoskeletal and other age-related diseases: a study protocol. Environ Health Prev Med. 2018 Jun;23(1):28. 
Watanabe et al.: Urinary Protein Profile of AD Patients

Griffin NM, Yu J, Long F, Oh P, Shore S, Li Y, et al. Label-free, normalized quantification of complex mass spectrometry data for proteomic analysis. Nat Biotechnol. 2010 Jan;28(1):83-9.

17 Huang W, Sherman BT, Lempicki RA. Bioinformatics enrichment tools: paths toward the comprehensive functional analysis of large gene lists. Nucleic Acids Res. 2009 Jan;37(1):1-13.

18 Huang W, Sherman BT, Lempicki RA. Systematic and integrative analysis of large gene lists using DAVID bioinformatics resources. Nat Protoc. 2009;4(1):44-57.

19 Sato H, Ishida S, Toda K, Matsuda R, Hayashi Y, Shigetaka M, et al. New approaches to mechanism analysis for drug discovery using DNA microarray data combined with KeyMolnet. Curr Drug Discov Technol. 2005 Jun; 2(2):89-98.

20 Filip S, Zoidakis J, Vlahou A, Mischak H. Advances in urinary proteome analysis and applications in systems biology. Bioanalysis. 2014;6(19):2549-69.

21 Marikanty RK, Gupta MK, Cherukuvada SV, Kompella SS, Prayaga AK, Konda S, et al. Identification of urinary proteins potentially associated with diabetic kidney disease. Indian J Nephrol. 2016 Nov-Dec;26(6):434-45.

22 Riaz S. Study of protein biomarkers of diabetes mellitus type 2 and therapy with bitamin B1. J Diabetes Res. 2015;2015:150176.

23 Siebert S, Porter D, Paterson C, Hampson R, Gaya D, Latosinska A, et al. Urinary proteomics can define distinct diagnostic inflammatory arthritis subgroups. Sci Rep. 2017 Jan;7(1):40473.

24 Kraus VB, Hargrove DE, Hunter DJ, Renner JB, Jordan JM. Establishment of reference intervals for osteoarthritis-related soluble biomarkers: the FNIH/OARSI OA Biomarkers Consortium. Ann Rheum Dis. 2017 Jan; 76(1):179-85.

25 Röthlisberger S, Pedroza-Diaz J: Urine protein biomarkers for detection of cardiovascular disease and their use for the clinic. Expert Rev Proteomics. 2017 Dec;14(12):1091-103.

26 Htun NM, Magliano DJ, Zhang ZY, Lyons J, Petit T, Nkuipou-Kenfack E, et al. Prediction of acute coronary syndromes by urinary proteome analysis. PLoS One. 2017 Mar;12(3):e0172036.

27 Husi H, Skipworth RJ, Cronshaw A, Fearon KC, Ross JA, Fearon KC, et al. Proteomic identification of potential cancer markers in human urine using subtractive analysis. Int J Oncol. 2016 May;48(5):1921-32.

28 Nolen BM, Lomakin A, Marrangoni A, Velikokhatnaya L, Prosser D, Lokshin AE. Urinary protein biomarkers in the early detection of lung cancer. Cancer Prev Res (Phila). 2015 Feb;8(2):111-9.

29 Wang W, Wang S, Zhang M. Identification of urine biomarkers associated with lung adenocarcinoma. Oncotarget. 2017 Jun;8(24):38517-29.

30 Bijnsdorp IV, Jimenez CR. Large-scale urinary proteome dataset across tumor types reveals candidate biomarkers for lung cancer. EBioMedicine. 2018 Apr;30:5-6.

31 Ruan Q, D'Onofrio G, Sancarlo D, Greco A, Yu Z. Potential fluid biomarkers for pathological brain changes in Alzheimer's disease: implication for the screening of cognitive frailty. Mol Med Rep. 2016 Oct;14(4):3184-98.

32 Yao F, Hong X, Li S, Zhang Y, Zhao Q, Du W, et al. Urine-Based Biomarkers for Alzheimer's Disease Identified Through Coupling Computational and Experimental Methods. J Alzheimers Dis. 2018 65(2):421-31.

33 Hirao Y, Saito S, Fujinaka H, Miyazaki S, Xu B, Quadery A, et al. Proteome profiling of diabetic mellitus patient urine for discovery of biomarkers by comprehensive MS-based proteomics. Proteomes. 2018 Feb;6(1):9.

34 Adachi J, Kumar C, Zhang Y, Olsen JV, Mann M. The human urinary proteome contains more than 1500 proteins, including a large proportion of membrane proteins. Genome Biol. 2006 7(9):R80.

35 Carmona-Gutierrez D, Hughes AL, Madeo F, Ruckenstuhl C. The crucial impact of lysosomes in aging and longevity. Ageing Res Rev. 2016 Dec;32:2-12.

36 Cataldo AM, Barnett JL, Berman SA, Li J, Quarless S, Bursztajn S, et al. Gene expression and cellular content of cathepsin D in Alzheimer's disease brain: evidence for early up-regulation of the endosomal-lysosomal system. Neuron. 1995 Mar;14(3):671-80.

37 Cataldo AM, Barnett JL, Mann DM, Nixon RA. Colocalization of lysosomal hydrolase and beta-amyloid in diffuse plaques of the cerebellum and striatum in Alzheimer's disease and Down's syndrome. J Neuropathol Exp Neurol. 1996 Jun;55(6):704-15.

38 Nixon RA, Mathews PM, Cataldo AM. The neuronal endosomal-lysosomal system in Alzheimer's disease. J Alzheimers Dis. 2001 Feb;3(1):97-107.

39 Gowrishankar S, Yuan P, Wu Y, Schrag M, Paradise S, Grutzendler J, et al. Massive accumulation of luminal protease-deficient axonal lysosomes at Alzheimer's disease amyloid plaques. Proc Natl Acad Sci USA. 2015 Jul; 112(28):E3699-708.

40 Emiliani C, Urbanelli L, Racanicchi L, Orlacchio A, Pelicci G, Sorbi S, et al. Up-regulation of glycohydrolases in Alzheimer's Disease fibroblasts correlates with Ras activation. J Biol Chem. 2003 Oct;278(40):38453-60.

41 Goetzl EJ, Boxer A, Schwartz JB, Abner EL, Petersen RC, Miller BL, et al. Altered lysosomal proteins in neuralderived plasma exosomes in preclinical Alzheimer disease. Neurology. 2015 Jul;85(1):40-7.

42 Yamashima T. Can "calpain-cathepsin hypothesis" explain Alzheimer neuronal death? Ageing Res Rev. 2016 Dec;32:169-79.

43 Stoka V, Turk V, Turk B. Lysosomal cathepsins and their regulation in aging and neurodegeneration. Ageing Res Rev. 2016 Dec;32:22-37.

44 Hook V, Funkelstein L, Wegrzyn J, Bark S, Kindy M, Hook G. Cysteine Cathepsins in the secretory vesicle produce active peptides: cathepsin $\mathrm{L}$ generates peptide neurotransmitters and cathepsin B produces betaamyloid of Alzheimer's disease. Biochim Biophys Acta. 2012 Jan;1824(1):89-104. 
45 Heppner FL, Ransohoff RM, Becher B. Immune attack: the role of inflammation in Alzheimer disease. Nat Rev Neurosci. 2015 Jun;16(6):358-72.

46 Wyss-Coray T, Rogers J. Inflammation in Alzheimer disease-a brief review of the basic science and clinical literature. Cold Spring Harb Perspect Med. 2012 Jan;2(1):a006346-006346.

47 Holmes C. Review: systemic inflammation and Alzheimer's disease. Neuropathol Appl Neurobiol. 2013 Feb; 39(1):51-68.

48 Le Page A, Dupuis G, Frost EH, Larbi A, Pawelec G, Witkowski JM, et al. Role of the peripheral innate immune system in the development of Alzheimer's disease. Exp Gerontol. 2018 Jul;107:59-66.

49 Hatting M, Tavares CD, Sharabi K, Rines AK, Puigserver P. Insulin regulation of gluconeogenesis. Ann N Y Acad Sci. 2018 Jan;1411(1):21-35.

50 Kandimalla R, Thirumala V, Reddy PH. Is Alzheimer's disease a Type 3 Diabetes? A critical appraisal. Biochim Biophys Acta Mol Basis Dis. 2017 May;1863(5):1078-89.

51 Zhang J, Chen C, Hua S, Liao H, Wang M, Xiong Y, et al. An updated meta-analysis of cohort studies: diabetes and risk of Alzheimer's disease. Diabetes Res Clin Pract. 2017 Feb;124:41-7.

52 Cheng G, Huang C, Deng H, Wang H. Diabetes as a risk factor for dementia and mild cognitive impairment: a meta-analysis of longitudinal studies. Intern Med J. 2012 May;42(5):484-91.

53 Rajpathak SN, He M, Sun Q, Kaplan RC, Muzumdar R, Rohan TE, et al. Insulin-like growth factor axis and risk of type 2 diabetes in women. Diabetes. 2012 Sep;61(9):2248-54.

54 Aneke-Nash CS, Xue X, Qi Q, Biggs ML, Cappola A, Kuller L, et al. The Association Between IGF-I and IGFBP-3 and Incident Diabetes in an Older Population of Men and Women in the Cardiovascular Health Study. J Clin Endocrinol Metab. 2017 Dec;102(12):4541-7.

55 Johansson P, Åberg D, Johansson JO, Mattsson N, Hansson O, Ahrén B, et al. Serum but not cerebrospinal fluid levels of insulin-like growth factor-I (IGF-I) and IGF-binding protein-3 (IGFBP-3) are increased in Alzheimer's disease. Psychoneuroendocrinology. 2013 Sep;38(9):1729-37.

$56 \mathrm{Hu}$ X, Yang Y, Gong D. Circulating insulin-like growth factor 1 and insulin-like growth factor binding protein-3 level in Alzheimer's disease: a meta-analysis. Neurol Sci. 2016 Oct;37(10):1671-7.

57 Iturria-Medina Y, Sotero RC, Toussaint PJ, Mateos-Pérez JM, Evans AC, Initiative TA, et al.; Alzheimer's Disease Neuroimaging Initiative. Early role of vascular dysregulation on late-onset Alzheimer's disease based on multifactorial data-driven analysis. Nat Commun. 2016 Jun;7(1):11934.

58 Rafieian-Kopaei M, Setorki M, Doudi M, Baradaran A, Nasri H. Atherosclerosis: process, indicators, risk factors and new hopes. Int J Prev Med. 2014 Aug;5(8):927-46.

59 Tinkov AA, Bjørklund G, Skalny AV, Holmgren A, Skalnaya MG, Chirumbolo S, et al. The role of the thioredoxin/ thioredoxin reductase system in the metabolic syndrome: towards a possible prognostic marker? Cell Mol Life Sci. 2018 May; 75(9):1567-86.

60 Madrigal-Matute J, Fernandez-Garcia CE, Blanco-Colio LM, Burillo E, Fortuño A, Martinez-Pinna R, et al. Thioredoxin-1/peroxiredoxin-1 as sensors of oxidative stress mediated by NADPH oxidase activity in atherosclerosis. Free Radic Biol Med. 2015 Sep;86:352-61.

61 Johnson JL. Metalloproteinases in atherosclerosis. Eur J Pharmacol. 2017 Dec;816:93-106.

62 Ou JR, Tan MS, Xie AM, Yu JT, Tan L. Heat shock protein 90 in Alzheimer's disease. BioMed Res Int. 2014;2014: 796869.

$63 \mathrm{Xu}$ Q, Metzler B, Jahangiri M, Mandal K. Molecular chaperones and heat shock proteins in atherosclerosis. Am J Physiol Heart Circ Physiol. 2012 Feb;302(3):H506-14.

64 Balzan S, Lubrano V. LOX-1 receptor: A potential link in atherosclerosis and cancer. Life Sci. 2018 Apr;198: 79-86.

65 Wang ZT, Zhong XL, Tan MS, Wang HF, Tan CC, Zhang W, et al. Association of lectin-like oxidized low density lipoprotein receptor 1 (OLR1) polymorphisms with late-onset Alzheimer disease in Han Chinese. Ann Transl Med. 2018 May;6(10):172.

66 Boom A, Pochet R, Authelet M, Pradier L, Borghgraef P, Van Leuven F, et al. Astrocytic calcium/zinc binding protein S100A6 over expression in Alzheimer's disease and in PS1/APP transgenic mice models. Biochim Biophys Acta. 2004 Dec;1742(1-3):161-8.

67 Lin Q, Cao Y, Gao J. Decreased expression of the APOA1-APOC3-APOA4 gene cluster is associated with risk of Alzheimer's disease. Drug Des Devel Ther. 2015 Sep;9:5421-31.

68 Kitamura K, Watanabe Y, Nakamura K, Sanpei K, Wakasugi M, Yokoseki A, et al. Modifiable Factors Associated with Cognitive Impairment in 1,143 Japanese Outpatients: The Project in Sado for Total Health (PROST). Dement Geriatr Cogn Disord Extra. 2016 Aug;6(2):341-9.

69 Vercruysse P, Vieau D, Blum D, Petersén Å, Dupuis L. Hypothalamic alterations in neurodegenerative diseases and their relation to abnormal energy metabolism. Front Mol Neurosci. 2018 Jan;11:2. 